\title{
Maternal blood glucose level and offspring glucose-insulin homeostasis: what is the role of offspring adiposity?
}

\author{
Ellen C. Francis ${ }^{1,2}$ (D) $\cdot$ Dana Dabelea ${ }^{1,2,3}$ (D) $\cdot$ Brandy M. Ringham ${ }^{1} \cdot$ Katherine A. Sauder $^{1,2,3}$ (D) Wei Perng $^{1,2}$ (D)
}

Received: 20 May 2020 / Accepted: 14 August 2020 / Published online: 6 October 2020

(C) Springer-Verlag GmbH Germany, part of Springer Nature 2020

\begin{abstract}
Aims/hypothesis The aim of this work was to investigate the association of maternal $\mathrm{HbA}_{1 \mathrm{c}}$ during mid-pregnancy with biomarkers of glucose-insulin homeostasis during early childhood (4-7 years of age) and to assess whether and how offspring adiposity at birth and at age 4-7 years mediates this relationship among 345 mother-child pairs in the Healthy Start Study. Methods The exposure was maternal $\mathrm{HbA}_{1 \mathrm{c}}(\mathrm{mmol} / \mathrm{mol})$ measured at 20-34 gestational weeks and categorised into tertiles. The outcomes were offspring fasting glucose, 1/insulin, HOMA2-IR, and HOMA2-B at age 4-7 years. The mediators were per cent fat mass (\%FM) at birth, \%FM at age 4-7 years, and the sum of the two as a metric of cumulative adiposity. Mediation analyses were conducted via a counterfactual-based approach. All models accounted for maternal race/ethnicity, offspring age and sex. Results There was a significant total effect of maternal $\mathrm{HbA}_{1 \mathrm{c}}$ on offspring glucose and 1/insulin. Specifically, we observed a positive trend across tertiles of $\mathrm{HbA}_{1 \mathrm{c}}$ and offspring glucose $(p$ trend $<0.001)$, and an inverse trend across tertiles of $\mathrm{HbA}_{1 \mathrm{c}}$ and offspring 1 /insulin ( $p$ trend $=0.04$ ). For instance, compared with offspring of women in the lowest tertile of $\mathrm{HbA}_{1 \mathrm{c}}$, those whose mothers were in the second and third tertiles had $0.04 \mathrm{mmol} / 1$ (95\% CI $-0.05,0.13)$ and $0.17 \mathrm{mmol} / \mathrm{l}(95 \% \mathrm{CI} 0.08,0.26)$ higher fasting glucose concentrations at age 4-7 years, respectively. Adjustment for pre-pregnancy BMI did not appreciably change the results. We found no evidence of mediation by offspring adiposity at any life stage.

Conclusions/interpretation Offspring of women with higher $\mathrm{HbA}_{1 \mathrm{c}}$ during pregnancy had higher fasting glucose and lower insulin sensitivity by early childhood. These relationships were largely unaffected by the child's own adiposity.
\end{abstract}

Keywords Childhood glucose metabolism $\cdot$ Lifecourse epidemiology $\cdot$ Maternal glycaemic control $\cdot$ Pregnancy

\begin{abstract}
Abbreviations
ADP Air displacement plethysmography

FFM Fat-free mass

$\%$ FM Per cent fat mass

Electronic supplementary material The online version of this article (https://doi.org/10.1007/s00125-020-05294-2) contains peer-reviewed but unedited supplementary material, which is available to authorised users.
\end{abstract}

Ellen C. Francis

Ellen.Francis@CUAnschutz.edu

1 Lifecourse Epidemiology of Adiposity and Diabetes (LEAD) Center, University of Colorado Denver Anschutz Medical Campus, Aurora, CO, USA

2 Department of Epidemiology, Colorado School of Public Health, University of Colorado Denver Anschutz Medical Campus, Aurora, CO, USA

3 Department of Pediatrics, School of Medicine, University of Colorado Denver Anschutz Medical Campus, Aurora, CO, USA

$\begin{array}{ll}\text { GDM } & \text { Gestational diabetes mellitus } \\ \text { HAPO-FUS } & \text { Hyperglycemia and Adverse } \\ & \text { Pregnancy Outcomes Follow-up Study }\end{array}$

\section{Introduction}

Several studies show a dose-response relationship between maternal blood glucose levels (even below gestational diabetes mellitus [GDM] diagnostic criteria) and offspring adiposity starting at birth $[1,2]$ and biomarkers of glucose-insulin homeostasis as early as 7 years of age $[3,4]$. Given the cooccurrence of excess adiposity and alterations in glucoseinsulin homeostasis, it is challenging to disentangle how blood glucose levels during pregnancy influence offspring metabolism independent of adiposity. Further, the extent to which the relationship between maternal blood glucose and offspring metabolism is mediated by adiposity at a specific life 


\section{Research in context}

What is already known about this subject?

- Maternal glycaemic status during pregnancy is associated with offspring adiposity and metabolic health

- Higher offspring adiposity is associated with perturbed glucose-insulin homeostasis

What is the key question?

- Does offspring adiposity at birth and/or in early childhood (4-7 years of age) mediate the association between maternal blood glucose level and offspring glucose-insulin homeostasis and, if so, does the mediation follow a sensitive or accumulation-of-risk lifecourse epidemiological model?

What are the new findings?

- Higher maternal $\mathrm{HbA}_{1 \mathrm{c}}$ is associated with higher fasting glucose and lower insulin sensitivity in offspring at age 4-7 years

- The relationship between maternal $\mathrm{HbA}_{1 \mathrm{c}}$ and offspring metabolic biomarkers is not mediated by offspring adiposity at birth, at age 4-7 years, or cumulatively from birth through early childhood

How might this impact on clinical practice in the foreseeable future?

- Understanding how adiposity mediates risk at different time points will inform optimal timing of interventions

stage (e.g. infancy vs childhood) or cumulatively over time remains ambiguous.

Two recent studies from the Hyperglycemia and Adverse Pregnancy Outcomes [5] Follow-up Study (HAPO-FUS) investigated associations between maternal blood glucose and offspring metabolic biomarkers independent of adiposity $[3,6]$. In both analyses, the association between maternal blood glucose and offspring biomarkers at 7-14 years of age was robust to adjustment for offspring BMI or sum of skinfolds, notwithstanding some slight attenuation in the estimate of interest, suggesting minimal mediation by offspring adiposity in late childhood and adolescence. Given that development of metabolic risk likely transpires from chronic excess adiposity, the dynamic changes in fat accrual across infancy and early childhood, and that these life stages are sensitive periods for development of obesity-related diseases, it is important to assess the role of adiposity during multiple life stages and consider the impact of cumulative adiposity over time.

In the present study, we close gaps in current literature by investigating the association of maternal $\mathrm{HbA}_{1 \mathrm{c}}$ during mid-tolate pregnancy with fasting biomarkers of glucose-insulin homeostasis during early childhood (age 4-7 years). Use of $\mathrm{HbA}_{1 \mathrm{c}}$ provides an assessment of blood glucose control over the prior 3-4 months, which is likely more relevant to offspring metabolism than acute assessments of maternal blood glucose levels. In the current study, $\mathrm{HbA}_{1 \mathrm{c}}$ was assessed at 27 gestational weeks to capture early- to mid-pregnancy maternal glucose metabolism, which may be a sensitive gestational period for metabolic programming given that divergences in fetal growth trajectories become apparent at around 20 weeks
[7] and that the maternal metabolic milieu during the first and second trimester is more strongly associated with offspring adiposity and metabolic risk than later in pregnancy [8].

In addition, we are interested in assessing whether offspring adiposity at birth and at 4-7 years of age may mediate the association of interest by way of two lifecourse epidemiological conceptual models: sensitive periods and accumulation of risk [9]. The conceptual model for testing these lifecourse models is depicted in Fig. 1. We hypothesised that higher maternal $\mathrm{HbA}_{1 \mathrm{c}}$ is positively associated with glucose concentrations in offspring during early childhood (age 47 years) and that this relationship is mediated to the largest extent by cumulative adiposity.

\section{Methods}

Study population Study participants were from the Healthy Start Study, a prospective longitudinal cohort of 1410 racially/ ethnically diverse pregnant women who were enrolled at $\leq 24$ gestational weeks from prenatal clinics at the University of Colorado Hospital between 2009 and 2014 [10, 11]. In brief, women completed in-person research visits during pregnancy. Following delivery, air displacement plethysmography (ADP) and anthropometric measurements were taken on neonates prior to discharge. When offspring were 4-7 years of age, they returned for an in-person visit during which an assessment of body composition via ADP, anthropometry and a fasting blood sample were obtained. All mothers provided written informed consent and children 7 years of age or older provided written 
Percentage of mediation

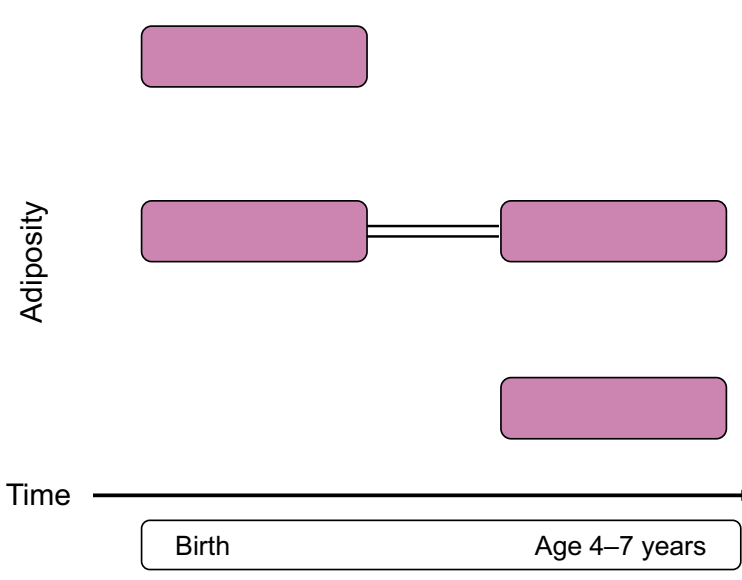

Fig. 1 Testing mediation by accumulation or sensitive-period effects of adiposity in models of the association between maternal $\mathrm{HbA}_{1 \mathrm{c}}$ in pregnancy and offspring glucose-insulin homeostasis in early childhood (age 4-7 years). Purple boxes represent adiposity either at a single point in time or the accumulation of adiposity over time. Sensitive-period model: during specific time periods (i.e. at age $4-7$ years), the mediation by adiposity on the relationship of interest has a stronger effect than it would at other times [18]. Thus, if mediation by offspring adiposity follows a

assent. The study protocol and procedures were approved by the Colorado Multiple Institutional Review Board.

Of the offspring of the 1410 women enrolled in the study, 778 attended an in-person child visit at age 4-7 years. We excluded 77 women who did not have data on $\mathrm{HbA}_{1 \mathrm{c}}$ during pregnancy and 87 offspring who did not have per cent fat mass (\%FM) measurements at birth. We further excluded 263 mother-child pairs without data on offspring adiposity and metabolic biomarkers at age 4-7 years, followed by six children who had \%FM values less than $4.5 \%$ and whose study record indicated movement during ADP measurement, yielding an analytic sample of 345 mother-child pairs (Fig. 2). We compared the maternal and offspring characteristics of those included vs excluded. The two samples were generally similar, except women in the analytical sample were $\sim 1.1$ years older than women excluded, and offspring were $\sim 76.2 \mathrm{~g}$ heavier at birth and had $\sim 1.2 \%$ higher fat mass at $4-7$ years of age.

\section{Exposure: maternal blood glucose levels during pregnancy At} median 17 (range 11-20) and 27 (range 20-34) gestational weeks, fasting blood was drawn from the women. Our primary exposure of interest was $\mathrm{HbA}_{1 \mathrm{c}}$, assayed from blood collected at 27 weeks, measured using a potassium ferricyanide assay by DCA Vantage Analyzer (Siemens, USA). Our secondary exposure was glucose concentrations measured at both time points using manufacturer pre-packaged enzymatic kits and the AU400e Chemistry Analyzer (Olympus America, USA).

Outcomes: biomarkers of offspring glucose-insulin homeostasis Fasting glucose was measured using manufacturer pre-

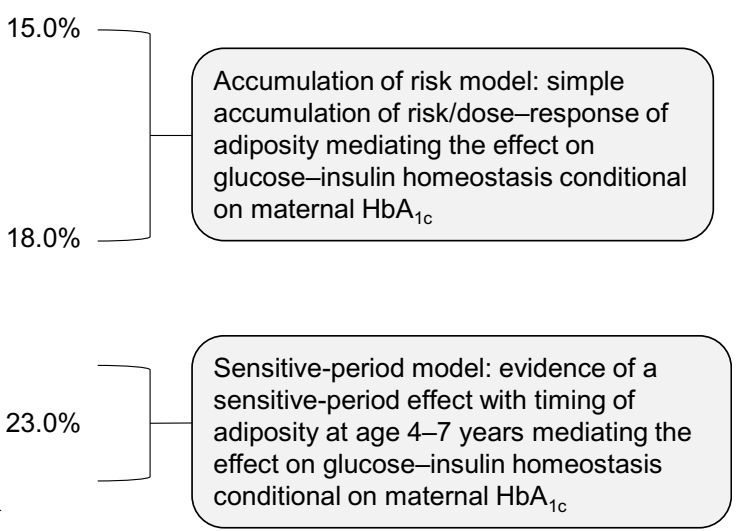

sensitive-period model, we would expect the magnitude of attenuation to be substantially greater at a specific life stage vs other time points. Accumulation of risk model: the accumulation of adiposity mediates the relationship of interest and the mediating effect of accumulated adiposity is greater than the mediating effect of adiposity at any one time-point. Adapted from Ben-Shlomo et al [9], under the terms of a CC BY 4.0 Attribution License (http://creativecommons.org/licenses/by/4.0/)

packaged enzymatic kits and the AU400e Chemistry Analyzer (Olympus America), and insulin was measured using an RIA by Millipore Corporation (USA). We calculated 1/(fasting insulin) as a measure of insulin sensitivity [12] and calculated an updated HOMA (HOMA2-IR and HOMA2-B) [13].

Mediators: neonatal and early childhood adiposity Neonatal body composition, including fat mass and fat-free mass (FFM), was measured by PEA POD (Life Measurement, USA) using densitometric techniques based on ADP [14]. This technology assesses \%FM via the direct measurement of a participant's mass and volume. Mass is measured on an electronic scale. A chamber is used to measure volume by applying laws of gas to pressure changes in the volume of air in the enclosed chamber prior to and after the participant enters. Body density is computed from participants' mass and volume and inserted into a standard formula [15] for estimating \%FM [14]. Measurements for each participant were taken in triplicate and the average of the two closest measures was used for analyses. At the visit at age 4-7 years, fat mass and FFM were measured using whole-body air plethysmography (BodPod; Life Measurement) with the Pediatric Option [16]. For this analysis, we focused on \%FM as the adiposity measure of interest because it provides an estimate of fat mass relative to total mass. In addition to assessing \% FM at birth and at age 4-7 years, we took the sum across both time points to capture cumulative adiposity from birth through early childhood.

Covariates and background characteristics We calculated maternal pre-pregnancy BMI $\left(\mathrm{kg} / \mathrm{m}^{2}\right)$ based on prepregnancy weight obtained from medical records $(89 \%)$ or 


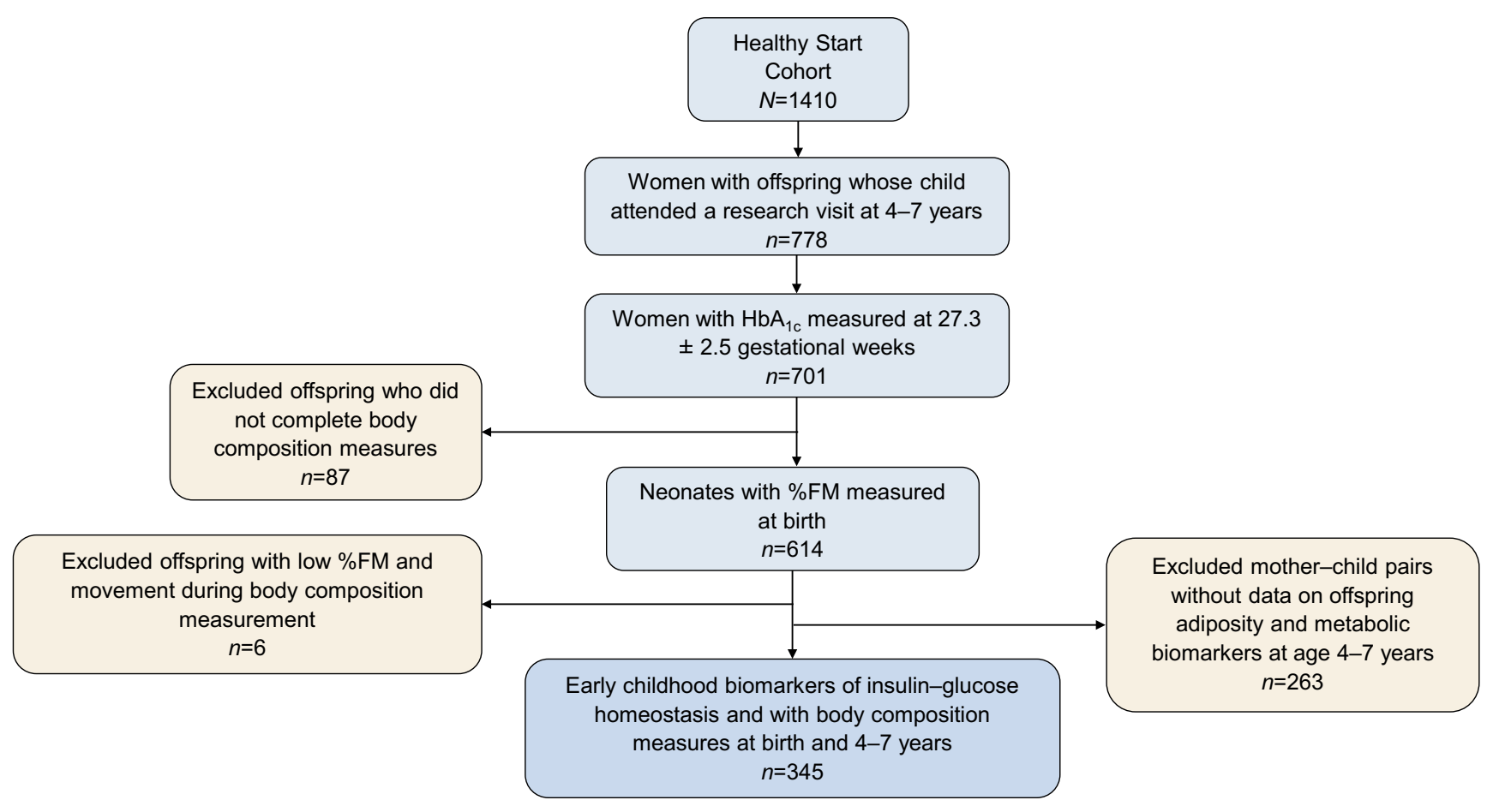

Fig. 2 Participant flow chart

self-report (11\%) and height measured at the first research visit. GDM status was determined based on medical record review. Maternal race/ethnicity, educational attainment, parity and smoking status during pregnancy were self-reported via questionnaire.

Among all offspring in the analytic sample, gestational age at birth was abstracted from medical records and offspring's weight and skinfold thickness were measured by trained nurses. Gestational age-specific birthweight $z$ scores were derived using US natality data as reference [17]. Triceps, subscapular and mid-thigh skinfolds were measured in triplicate and the two closest measurements were averaged. Skinfolds were summed (sum of skinfolds) as a measure of subcutaneous adiposity. At the visit at age 4-7 years, a child's age was calculated as the difference between date of the research visit and delivery date.

Data analysis Prior to formal analysis, we examined bivariate associations of maternal and offspring characteristics across tertiles of $\mathrm{HbA}_{1 \mathrm{c}}$. This step in conjunction with our prior knowledge informed selection of covariates, selection of potential confounders of the mediator-outcome relationship, and confounders of the mediator-outcome relationship that could be affected by exposure [18]. In the main analysis, we assessed $\mathrm{HbA}_{1 \mathrm{c}}$ continuously as well as in tertiles to allow for potential non-linear associations. We tested for a linear trend by introducing the three-level variable as a continuous indicator, with each level set to the median value of each tertile [19]. Due to multiple participants with the same $\mathrm{HbA}_{1 \mathrm{c}}$ value, the number of participants within each $\mathrm{HbA}_{1 \mathrm{c}}$ tertile was unequal, with 151 participants in tertile 1,98 in tertile 2 and 96 in tertile 3 . For this reason, we focus our interpretation on associations with respect to tertiles of $\mathrm{HbA}_{1 \mathrm{c}}$.

In the multivariable linear regression models, maternal $\mathrm{HbA}_{1 \mathrm{c}}$ (tertiles and per $1 \mathrm{mmol} / \mathrm{mol}$ ) was the exposure and offspring glucose, 1/insulin, HOMA2-IR and HOMA2-B were assessed as separate outcomes. Model 1 adjusted for maternal race/ethnicity and offspring sex, age and number of days between birth and neonatal ADP assessment to account for potential changes in body composition following delivery. Model 2 further accounted for maternal pre-pregnancy BMI as a sensitivity analysis given the overlap in developmental pathways linking higher BMI and blood glucose level during pregnancy to offspring outcome [20].

We conducted mediation analysis using a counterfactualbased approach [18], which allows for exposure-mediator interactions, though we did not observe such interactions in our data. The Directed Acyclic Graphs for the analysis are shown in Fig. 3. We estimated the total effect (i.e. overall effect) of the exposure-outcome association (path $\mathrm{C}$ in Fig. 3), the exposure-mediator association (path $\mathrm{A}$ in Fig. 3), and the mediator-outcome association (path B in Fig. 3). When the mediator is included as a covariate (\%FM at birth, age 4-7 years, or cumulative \%FM), the exposureoutcome association is referred to as the natural direct effect (path $\mathrm{C}^{\prime}$ in Fig. 3). Here, we interpret the natural direct effect as the effect of $\mathrm{HbA}_{1 \mathrm{c}}$ on offspring biomarkers that does not operate through offspring $\% \mathrm{FM}$. The magnitude of mediation 
a

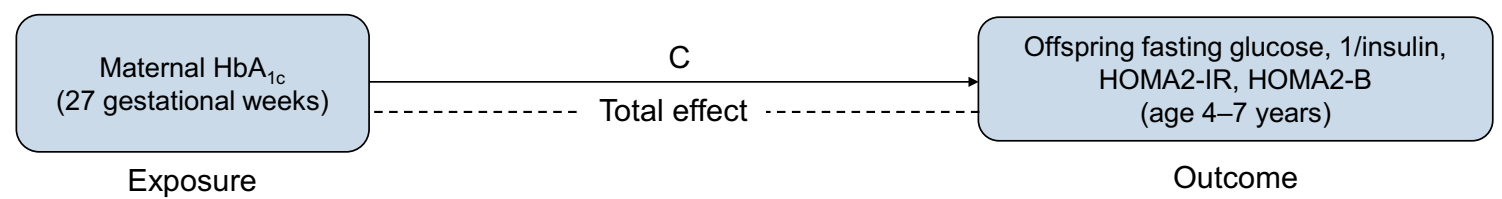

b

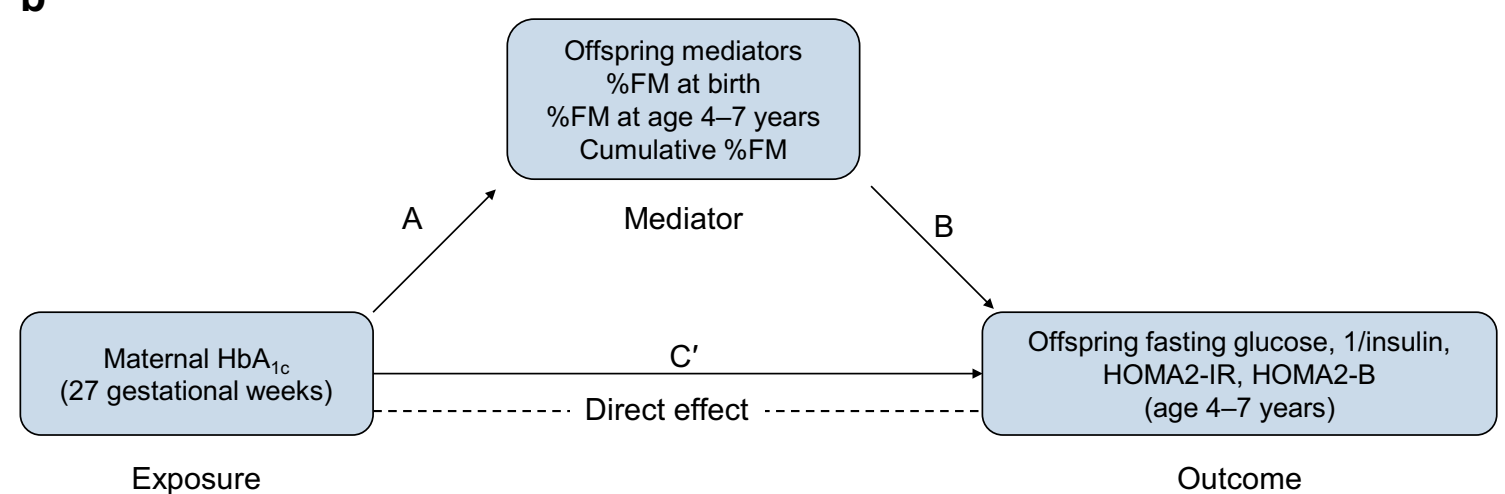

Fig. 3 Directed acyclic graphs of the relationships between maternal $\mathrm{HbA}_{1 \mathrm{c}}$, offspring adiposity and offspring biomarkers of insulin-glucose homeostasis. For the total effect of exposure-outcome relationship (a), the total effect is represented by path $\mathrm{C}$. For the direct effect in a mediation model (b), in which path A represents the exposure-mediator relationship and path $\mathrm{B}$ represents the mediator-outcome relationship, the

was determined by taking the difference between the total effect (path $\mathrm{C}$ in Fig. 3) and the natural direct effect (path $\mathrm{C}^{\prime}$ in Fig. 3) [18].

We tested two lifecourse epidemiological models to evaluate the role of $\% \mathrm{FM}$ as a mediator: the sensitive-period model, wherein exposure to risk factors during a specific life stage has long-term effects on disease risk; and the accumulation-of-risk model wherein risk factors for disease accumulate across the lifecourse and have additive effects on disease risk. To assess whether \%FM mediates the above associations via a sensitiveperiod or an accumulation-of-risk framework, we compared the natural direct effects between models that included \%FM at birth, at age 4-7 years, or the sum of \%FM across the two life stages as a metric of cumulative adiposity (Fig. 1). We hypothesised that if \%FM followed a sensitive-period model, then the magnitude of mediation would be greater at a specific life stage. Alternatively, if \%FM followed an accumulation-of risk-model, then inclusion of cumulative \%FM would result in greater mediation than inclusion of either life stage by itself. For all models, we tested for an interaction with maternal race/ethnicity and offspring sex and considered stratified analyses if $p$ for interaction was $<0.05$.

We performed sensitivity analyses to examine the robustness of our findings. First, rather than \%FM, we used offspring BMI as the indicator of adiposity [21] and assessed this variable as a mediator using the same steps described above for \%FM. Second, because maternal fasting glucose is direct effect is represented by path $\mathrm{C}^{\prime}$ and measures the effect of maternal $\mathrm{HbA}_{1 \mathrm{c}}$ on biomarkers of offspring glucose-insulin homeostasis that does not operate through (i.e. is independent of) offspring \%FM (the mediators). The difference between path $\mathrm{C}$ and path $\mathrm{C}^{\prime}$ represents the effect of $\mathrm{HbA}_{1 \mathrm{c}}$ on offspring biomarkers that operates through \% FM

a more commonly used metric of maternal blood glucose, we examined Pearson's correlations among $\mathrm{HbA}_{1 \mathrm{c}}$ with fasting glucose at 17 weeks (range 11-20) and at 27 weeks (range 2034 ) and assessed whether the associations of interest differed when maternal fasting glucose at either time point was the exposure. Third, we excluded cases of preterm delivery $(<37$ gestational weeks), pre-eclampsia $(n=12)$ and overt GDM $(n=14)$ to assess the impact of these complications on results.

All models met assumptions of multivariate normality. We considered two-sided $p$ values $<0.05$ to be statistically significant. SAS version 9.4 (SAS Institute, Cary, NC, USA) was used for all analyses.

\section{Results}

Characteristics The mean $\pm \mathrm{SD}$ age of the women at enrolment was $28.7 \pm 6.0$ years, most were non-Hispanic white $(58.6 \%)$ and the median (range) of maternal $\mathrm{HbA}_{1 \mathrm{c}}$ was $96.8 \mathrm{mmol} /$ mol (59.5-134.1) (5.0\% [3.7-6.1]). At the 4-7 years visit, the children were $4.8 \pm 0.7$ years of age and approximately half (46.7\%) were female. As expected, we observed differences in membership of $\mathrm{HbA}_{1 \mathrm{c}}$ tertiles by maternal race/ethnicity. For example, $70.9 \%$ of non-Hispanic white women were in the lowest tertile of $\mathrm{HbA}_{1 \mathrm{c}}$, as compared with $16.6 \%$ of Hispanic women and $9.9 \%$ of non-Hispanic black women ( $p$ for difference across all race/ethnic groups $<0.001$ ). We also observed an 
inverse relationship for education level $(p$ difference $<0.001)$ and a positive association for pre-pregnancy BMI ( $p$ trend $<0.001)$ with $\mathrm{HbA}_{1 \mathrm{c}}$. In terms of offspring characteristics, maternal $\mathrm{HbA}_{1 \mathrm{c}}$ was positively correlated with indicators of adiposity at birth and during early childhood (age 4-7 years), with more prominent associations in early childhood. Higher maternal $\mathrm{HbA}_{1 \mathrm{c}}$ corresponded with higher fasting glucose ( $p$ trend $=0.03$ ), HOMA2-IR ( $p$ trend $<0.0001)$ and $1 /$ (fasting insulin) ( $p$ trend $=0.01$ ) during early childhood. Maternal $\mathrm{HbA}_{1 \mathrm{c}}$ was not related to HOMA2-B. Additional participant characteristics are shown in Table 1.

Total effect of maternal $\mathrm{HbA}_{1 \mathrm{c}}$ and early childhood biomarkers of glucose-insulin homeostasis Table 2 shows total effects of maternal $\mathrm{HbA}_{1 \mathrm{c}}$ on the metabolic biomarkers in offspring. In Model 1, which accounted for maternal race/ethnicity and child's age and sex, we observed a positive linear trend across tertiles of $\mathrm{HbA}_{1 \mathrm{c}}$ in relation to offspring fasting glucose $(p$ trend $<0.001)$. Specifically, offspring of women in the second and third tertiles of $\mathrm{HbA}_{1 \mathrm{c}}$ had glucose concentrations that were $0.04 \mathrm{mmol} / \mathrm{l}(95 \% \mathrm{CI}-0.05,0.13)$ and $0.17 \mathrm{mmol} / 1$ (95\% CI 0.08, 0.26), respectively, higher than those of women in the lowest tertile. We also found an inverse association for $\mathrm{HbA}_{1 \mathrm{c}}$ tertiles and offspring 1/(fasting insulin) $(p$ trend $=0.04)$. Adjustment for pre-pregnancy BMI in Model 2 did not change these results, although the upper confidence limit for $1 /$ (fasting insulin) crossed the null.

Assessment of offspring adiposity as mediator Estimates of total effects and natural direct effects after inclusion of \%FM at birth, at age 4-7 years and cumulatively across both life stages can be found in Table 3 . Maternal $\mathrm{HbA}_{1 \mathrm{c}}$ was positively associated with offspring \% FM at both life stages individually and cumulatively (electronic supplementary material [ESM] Table 1), and all three metrics of \%FM were associated with $1 /$ (fasting insulin), HOMA-IR and HOMA-B, but not fasting glucose (ESM Table 2). Adjusting for offspring $\% \mathrm{FM}$ at any time point did not appreciably attenuate the association of $\mathrm{HbA}_{1 \mathrm{c}}$ with offspring biomarkers. After exploring different indicators of $\% \mathrm{FM}$, there was no indication that offspring \%FM played a mediating role that followed the sensitive-period model or the accumulation-of-risk model.

To illustrate this, when comparing the total effect of the third tertile vs first tertile of $\mathrm{HbA}_{1 \mathrm{c}}$ in relation to offspring glucose $(0.17 \mathrm{mmol} / \mathrm{l}[95 \% \mathrm{CI} 0.08,0.26])$, we observed minimal differences between this estimate and the natural direct effect after including \%FM at birth $(0.17 \mathrm{mmol} / 1[0.08,0.27] ; 0 \%$ change $)$, $\% \mathrm{FM}$ at $4-7$ years $(0.16 \mathrm{mmol} / 1[0.07,0.26] ; 6 \%$ decrease $)$, and cumulative $\% \mathrm{FM}(0.17 \mathrm{mmol} / 1[0.08,0.26] ; 0 \%$ change $)$. Similarly, there were minimal differences between the natural direct effects and total direct effect for $1 /$ (fasting insulin).

In all models, we noted similar but largely non-significant associations when continuous maternal $\mathrm{HbA}_{1 \mathrm{c}}$ was the exposure of interest. This is likely due to the fact that, despite having a relatively normal distribution and meeting assumptions of multivariate normality, several women in our sample had a similar $\mathrm{HbA}_{1 \mathrm{c}}\left(n=98\right.$ [28.4\%] with $\mathrm{HbA}_{1 \mathrm{c}}=$ $96.8 \mathrm{mmol} / \mathrm{mol}$ [5.1\%] or $99.7 \mathrm{mmol} / \mathrm{mol}$ [5.0\%]), thereby reducing variability in the explanatory variable.

Sensitivity analyses In sensitivity analyses, we observed similar findings when using offspring BMI as a mediator and when maternal fasting glucose at 17 or 27 weeks was the exposure (ESM Table 3), which aligns with the finding that $\mathrm{HbA}_{1 \mathrm{c}}$ was significantly positively correlated to maternal fasting glucose (ESM Table 4). We noted a statistical interaction between $\mathrm{HbA}_{1 \mathrm{c}}$ and race/ethnicity for some of the offspring biomarkers but did not observe different associations in stratified models. Therefore, final models are among all race/ethnic groups. The magnitude and direction of associations were unchanged after excluding individuals experiencing preterm delivery, pre-eclampsia and GDM; thus we included these individuals in the study sample.

\section{Discussion}

In this prospective study of 345 racially/ethnically diverse US mother-offspring pairs, higher maternal early- to midpregnancy $\mathrm{HbA}_{1 \mathrm{c}}$, even below diagnostic levels for diabetes, corresponded with higher offspring fasting glucose levels and lower insulin sensitivity (1/[fasting insulin]) by 4-7 years of age, even after accounting for maternal pre-pregnancy BMI. Offspring adiposity at birth or at age 4-7 years, or cumulatively across the two time points, did not play a substantial role in mediating the effect of maternal $\mathrm{HbA}_{1 \mathrm{c}}$ on metabolic biomarkers in offspring. These findings suggest the existence of alternative pathways through which maternal blood glucose levels may impact offspring glucose-insulin homeostasis.

Maternal $\mathrm{HbA}_{1 \mathrm{c}}$ is associated with biomarkers of glucoseinsulin homeostasis in offspring The total effect of maternal $\mathrm{HbA}_{1 \mathrm{c}}$ on metabolic biomarkers in offspring was most evident for fasting glucose and $1 /$ (fasting insulin), a biomarker of insulin sensitivity. This is consistent with findings from a small historical cohort of 89 mother-child dyads [22], a sample of 514 mother-offspring pairs in India [23], and an analysis among 4160 HAPO-FUS participants [24]. However, these previous studies focused on in utero exposure to overt maternal diabetes [23] or GDM [22, 24], whereas we were able to detect effects of maternal blood glucose levels on offspring metabolic biomarkers in a cohort of predominantly GDM-free women. The positive association between maternal $\mathrm{HbA}_{1 \mathrm{c}}$ and offspring glucose, in conjunction with the inverse association with $1 /$ (fasting insulin), could indicate diminished capacity to maintain glucose-insulin homeostasis among 
Table 1 Bivariate associations of maternal and offspring characteristics with maternal $\mathrm{HbA}_{1 \mathrm{c}}$

\begin{tabular}{|c|c|c|c|c|c|}
\hline \multirow[t]{2}{*}{ Characteristic } & \multirow[t]{2}{*}{ Overall $(n=345)$} & \multicolumn{3}{|l|}{$\mathrm{HbA}_{1 \mathrm{c}}$} & \multirow[t]{2}{*}{$p$ value } \\
\hline & & Tertile 1 & Tertile 2 & Tertile 3 & \\
\hline \multicolumn{6}{|l|}{ Maternal characteristics } \\
\hline $\mathrm{HbA}_{1 \mathrm{c}}, \mathrm{mmol} / \mathrm{mol}$, median (range) & $96.8(59.5-134.1)$ & $89.4(59.5-93.9)$ & $98.2(96.8-99.7)$ & $107.5(102.5-134.1)$ & \\
\hline $\mathrm{HbA}_{1 \mathrm{c}}, \%$, median (range) & $5.0(3.7-6.1)$ & $4.8(3.7-4.9)$ & $5.1(5.0-5.1)$ & $5.3(5.2-6.1)$ & - \\
\hline $\mathrm{GA}$ at $\mathrm{HbA}_{1 \mathrm{c}}$ measurement, weeks & $27 \pm 2.4$ & $26.4 \pm 2.2$ & $27.3 \pm 2.4$ & $27.8 \pm 2.3$ & $<0.001$ \\
\hline Age at the first pregnancy visit, years & $28.7 \pm 6.0$ & $28.5 \pm 5.9$ & $29.4 \pm 6$ & $28.2 \pm 6.2$ & 0.76 \\
\hline Race/ethnicity & & & & & $<0.001$ \\
\hline Non-Hispanic white & $202(58.6)$ & $107(70.9)$ & $60(61.2)$ & $35(36.5)$ & \\
\hline Non-Hispanic black & $52(15.1)$ & $15(9.9)$ & $15(15.3)$ & $22(22.9)$ & \\
\hline Hispanic & $74(21.5)$ & $25(16.6)$ & $20(20.4)$ & $29(30.2)$ & \\
\hline Other $^{\mathrm{a}}$ & $17(4.9)$ & $4(2.6)$ & $3(3.1)$ & $10(10.4)$ & \\
\hline Education & & & & & $<0.001$ \\
\hline High school or less & $94(27.2)$ & $32(21.2)$ & $24(24.5)$ & $38(39.6)$ & \\
\hline Some college/associates degree & 79 (22.9) & $28(18.5)$ & $26(26.5)$ & $25(26.0)$ & \\
\hline College graduate & 79 (22.9) & $40(26.5)$ & $24(24.5)$ & $15(15.6)$ & \\
\hline Graduate degree & $93(27.0)$ & $51(33.8)$ & $24(24.5)$ & $18(18.8)$ & \\
\hline Nulliparous & $160(46.4)$ & $74(49.0)$ & $55(56.1)$ & $56(58.3)$ & 0.13 \\
\hline Smoked during pregnancy & $25(7.2)$ & $9(5.9)$ & $6(6.2)$ & $10(10.4)$ & 0.21 \\
\hline Pre-pregnancy BMI, $\mathrm{kg} / \mathrm{m}^{2}$ & $25.9 \pm 6.1$ & $24.7 \pm 5$ & $26.5 \pm 7.3$ & $27.2 \pm 6.0$ & $<0.001$ \\
\hline Gestational weight gain ${ }^{\mathrm{b}}$ & & & & & 0.09 \\
\hline Insufficient & $56(16.4)$ & $21(14.1)$ & $10(10.2)$ & $25(26.3)$ & \\
\hline Adequate & $47(13.7)$ & $23(15.4)$ & $14(14.3)$ & $10(10.5)$ & \\
\hline Excessive & $239(69.9)$ & $105(70.5)$ & $74(75.5)$ & $60(63.2)$ & \\
\hline \multicolumn{6}{|l|}{ Offspring characteristics } \\
\hline Female sex & $161(46.7)$ & $79(52.3)$ & $39(40.2)$ & $39(44.3)$ & 0.12 \\
\hline Race/ethnicity & & & & & $<0.001$ \\
\hline Non-Hispanic white & $185(53.6)$ & $95(62.9)$ & $54(55.1)$ & $36(37.5)$ & \\
\hline Non-Hispanic black & $43(12.5)$ & $14(9.3)$ & $9(9.2)$ & $20(20.8)$ & \\
\hline Hispanic & $76(22.0)$ & $26(17.2)$ & $21(21.4)$ & $29(30.2)$ & \\
\hline Other ${ }^{\mathrm{a}}$ & $41(11.9)$ & $16(10.6)$ & $14(14.3)$ & $11(11.5)$ & \\
\hline \multicolumn{6}{|l|}{ Neonatal } \\
\hline GA at delivery, weeks & $39.5 \pm 1.2$ & $39.6 \pm 1.2$ & $39.5 \pm 1.2$ & $39.4 \pm 1.4$ & 0.18 \\
\hline Birthweight, $g$ & $3166 \pm 426$ & $3147 \pm 388$ & $3158 \pm 413$ & $3205 \pm 491$ & 0.30 \\
\hline Birthweight-for-gestational age $z$ score ${ }^{\mathrm{c}}$ & $-0.6 \pm 0.9$ & $-0.6 \pm 0.8$ & $-0.6 \pm 0.9$ & $-0.4 \pm 0.9$ & 0.06 \\
\hline Fat mass, $\mathrm{kg}$ & $0.3 \pm 0.1$ & $0.3 \pm 0.1$ & $0.3 \pm 0.1$ & $0.3 \pm 0.2$ & 0.06 \\
\hline$\% \mathrm{FM}$ & $9.5 \pm 3.8$ & $9.2 \pm 3.6$ & $9.4 \pm 3.9$ & $10.0 \pm 4.0$ & 0.11 \\
\hline Sum of skinfolds, mm & $15.3 \pm 3.7$ & $15.3 \pm 3.3$ & $15.1 \pm 3.8$ & $15.5 \pm 4.1$ & 0.73 \\
\hline \multicolumn{6}{|l|}{ Early childhood } \\
\hline Age, years & $4.8 \pm 0.7$ & $4.7 \pm 0.6$ & $4.8 \pm 0.8$ & $4.9 \pm 0.7$ & 0.06 \\
\hline $\mathrm{BMI}, \mathrm{kg} / \mathrm{m}^{2}$ & $15.6 \pm 1.5$ & $15.4 \pm 1.4$ & $15.6 \pm 1.3$ & $15.8 \pm 1.7$ & 0.04 \\
\hline BMI-for-age $z$ score $^{\mathrm{d}}$ & $0.2 \pm 1.0$ & $0.1 \pm 0.9$ & $0.2 \pm 0.9$ & $0.3 \pm 1.1$ & 0.08 \\
\hline Fat mass, $\mathrm{kg}$ & $3.8 \pm 1.5$ & $3.5 \pm 1.3$ & $3.8 \pm 1.3$ & $4.1 \pm 1.7$ & $<0.001$ \\
\hline$\% \mathrm{FM}$ & $20.4 \pm 6.1$ & $19.9 \pm 6.1$ & $20.3 \pm 6$ & $21.1 \pm 6.2$ & 0.15 \\
\hline Sum of skinfolds, mm & $32.5 \pm 9.5$ & $31.2 \pm 8.8$ & $33.1 \pm 9.4$ & $33.8 \pm 10.5$ & 0.03 \\
\hline Glucose, $\mathrm{mmol} / \mathrm{l}$ & $4.6 \pm 0.4$ & $4.5 \pm 0.4$ & $4.6 \pm 0.3$ & $4.7 \pm 0.4$ & 0.03 \\
\hline HOMA2-IR ${ }^{\mathrm{e}}$ & $0.8 \pm 0.4$ & $0.75 \pm 0.37$ & $0.81 \pm 0.41$ & $0.89 \pm 0.39$ & $<0.001$ \\
\hline HOMA2-B $^{\mathrm{e}}$ & $96.9 \pm 27.0$ & $95.2 \pm 27.4$ & $97.1 \pm 26.9$ & $98.5 \pm 26.6$ & 0.43 \\
\hline 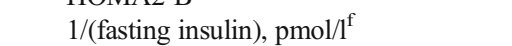 & $0.03 \pm 0.02$ & $0.03 \pm 0.01$ & $0.03 \pm 0.02$ & $0.03 \pm 0.01$ & 0.01 \\
\hline
\end{tabular}

Data are presented as mean $\pm \mathrm{SD}$ or $n(\%)$ unless stated otherwise. Not all characteristics were observed within the 345 mother-offspring pairs; therefore, percentages represent the distribution among women or offspring with available data for the specific characteristic

${ }^{a}$ Other includes Asian, American Indian/Alaska natives, Hawaiian/Pacific Islanders and multiracial

${ }^{\mathrm{b}}$ Gestational weight gain categories classified according to the Institute of Medicine 2009 guidelines [42]

${ }^{\mathrm{c}}$ Birthweight $z$ score specific to gestational age derived from US natality reference [17]

${ }^{\mathrm{d}}$ BMI specific to age derived from WHO growth standards [43]

${ }^{\mathrm{e}}$ Updated HOMA calculated using a computer program [13]

${ }^{\mathrm{f}}$ A measure of insulin sensitivity [12]

$p$ values represent type III main effects for categorical variables and are shown as $p$ trend for ordinal variables test for significant differences across $\mathrm{HbA}$ tertiles based on generalised linear models

GA, gestational age 
Table 2 Total effect of maternal $\mathrm{HbA}_{1 \mathrm{c}}$ on offspring biomarkers of glucose-insulin homeostasis at age $4-7$ years

\begin{tabular}{|c|c|c|c|c|}
\hline \multirow[t]{2}{*}{ Biomarker } & \multicolumn{2}{|l|}{ Model 1} & \multicolumn{2}{|l|}{ Model 2} \\
\hline & $\beta(95 \% \mathrm{CI})$ & $p$ value & $\beta(95 \% \mathrm{CI})$ & $p$ value \\
\hline \multicolumn{5}{|l|}{ Glucose, mmol/l } \\
\hline $\mathrm{HbA}_{1 \mathrm{c}} \mathrm{T} 2$ vs $\mathrm{T} 1$ & $0.04(-0.05,0.13)$ & & $0.04(-0.05,0.13)$ & \\
\hline $\mathrm{HbA}_{1 \mathrm{c}} \mathrm{T} 3 \mathrm{vs} \mathrm{T} 1$ & $0.17(0.08,0.26)^{*}$ & $<0.001^{\mathrm{a} *}$ & $0.16(0.06,0.25)^{*}$ & $<0.001^{\mathrm{a} *}$ \\
\hline $\mathrm{HbA}_{1 \mathrm{c}}($ per $1 \mathrm{mmol} / \mathrm{mol})$ & $0.003(-0.001,0.008)$ & $0.14^{\mathrm{b}}$ & $0.003(-0.002,0.007)$ & $0.23^{\mathrm{b}}$ \\
\hline \multicolumn{5}{|l|}{ 1/(fasting insulin), $\mathrm{pmol} / \mathrm{l}$} \\
\hline $\mathrm{HbA}_{1 \mathrm{c}} \mathrm{T} 2$ vs $\mathrm{T} 1$ & $-0.001(-0.004,0.003)$ & & $0.000(-0.004,0.003)$ & \\
\hline $\mathrm{HbA}_{1 \mathrm{c}} \mathrm{T} 3$ vs $\mathrm{T} 1$ & $-0.004(-0.008,-0.000)^{*}$ & $0.04^{\mathrm{a} *}$ & $-0.004(-0.008,0.000)$ & $0.06^{\mathrm{a}}$ \\
\hline $\mathrm{HbA}_{1 \mathrm{c}}($ per $1 \mathrm{mmol} / \mathrm{mol})$ & $-0.000(-0.000,-0.000)^{*}$ & $0.04^{\mathrm{b} *}$ & $-0.000(-0.000,0.000)$ & $0.07^{\mathrm{b}}$ \\
\hline \multicolumn{5}{|l|}{ HOMA2-IR } \\
\hline $\mathrm{HbA}_{1 \mathrm{c}} \mathrm{T} 2$ vs $\mathrm{T} 1$ & $0.05(-0.05,0.15)$ & & $0.04(-0.06,0.14)$ & \\
\hline $\mathrm{HbA}_{1 \mathrm{c}} \mathrm{T} 3$ vs $\mathrm{T} 1$ & $0.09(-0.00,0.20)$ & $0.08^{\mathrm{a}}$ & $0.08(-0.02,0.19)$ & $0.11^{\mathrm{a}}$ \\
\hline $\mathrm{HbA}_{1 \mathrm{c}}($ per $1 \mathrm{mmol} / \mathrm{mol})$ & $0.003(-0.002,0.008)$ & $0.21^{\mathrm{b}}$ & $0.002(-0.002,0.007)$ & $0.32^{\mathrm{b}}$ \\
\hline \multicolumn{5}{|l|}{ HOMA2-B } \\
\hline $\mathrm{HbA}_{1 \mathrm{c}} \mathrm{T} 2$ vs $\mathrm{T} 1$ & $1.10(-5.65,7.85)$ & & $0.80(-5.97,7.58)$ & \\
\hline $\mathrm{HbA}_{1 \mathrm{c}} \mathrm{T} 3 \mathrm{vs} \mathrm{T} 1$ & $0.99(-6.11,8.08)$ & $0.78^{\mathrm{a}}$ & $0.62(-6.52,7.76)$ & $0.86^{\mathrm{a}}$ \\
\hline $\mathrm{HbA}_{1 \mathrm{c}}($ per $1 \mathrm{mmol} / \mathrm{mol})$ & $0.175(-0.148,0.498)$ & $0.29^{\mathrm{b}}$ & $0.156(-0.170,0.483)$ & $0.35^{\mathrm{b}}$ \\
\hline \multicolumn{5}{|c|}{$\begin{array}{l}\text { Model } 1 \text { adjusted for maternal race/ethnicity, child's sex and age at assessment; Model } 2 \text { included the same } \\
\text { adjustments as Model 1, and was additionally adjusted for pre-pregnancy BMI }\end{array}$} \\
\hline \multicolumn{5}{|c|}{ a $p$ trend } \\
\hline \multicolumn{5}{|c|}{${ }^{\mathrm{b}} p$ difference } \\
\hline \multicolumn{5}{|c|}{$*$ Statistical significance at $\alpha=0.05$} \\
\hline $\mathrm{T} 1$, tertile $1 ; \mathrm{T} 2$, tertile $2 ; \mathrm{T} 3$, & ertile 3 & & & \\
\hline
\end{tabular}

offspring exposed to higher maternal blood glucose levels in utero. While modest, the differences we detected are noteworthy given that decreases in insulin sensitivity and mild increases in fasting glucose are precursors to development of diabetes [25, 26]. Our data suggest that differences in glucose-insulin homeostasis are detectable as early as 47 years of age following exposure to a spectrum of $\mathrm{HbA}_{1 \mathrm{c}}$ levels during gestation.

The effect of maternal $\mathrm{HbA}_{1 \mathrm{c}}$ on offspring insulin-glucose metabolism is not mediated by adiposity We did not find evidence for mediation by offspring adiposity at birth, at age 4-7 years, or cumulatively across both time points. While consistent with results of the aforementioned studies led by Scholtens et al [3] and Tam et al [6], these null findings are counter to our hypothesis, given that cumulative adiposity is a strong risk factor for dysregulated glucose-insulin homeostasis in children and adults [27, 28]. Regardless, adiposity tracks throughout the lifecourse [29] and metabolic diseases transpire from chronic (as opposed to acute) exposures over time. Therefore, as offspring age, cumulative adiposity may become a more biologically relevant mediator. In studies assessing longitudinal effects of GDM on offspring insulin and glucose, differences in these biomarkers with respect to GDM exposure became greater as the children approached puberty [23, 30].
Thus, we suspect that differences in early childhood glucoseinsulin homeostasis are likely to persist and become amplified later in life, with divergences in offspring metabolic trajectories proportional to the degree of maternal hyperglycaemia.

Potential mechanisms There are a few mechanisms that might link maternal hyperglycaemia to offspring glucose-insulin metabolism independent of offspring adiposity. Emerging data from human and animal studies suggest that chronic nutrient excess and beta cell overstimulation in early life lead to beta cell dysfunction, which later develops into hyperglycaemia [31]. Animal models also demonstrate that altered maternal insulin secretion and resistance directly alter fetal beta cell development, insulin secretion and insulin uptake by target tissues [32-34]. Additionally, we cannot rule out the effects of maternal adiposity on offspring metabolism, although in the present analysis, adjustment for maternal prepregnancy BMI did not change our findings. These mechanisms, either independently or jointly, may lead to unfavourable changes in development and function of key organs and tissues involved in glucose metabolism [34, 35].

Beyond fetal programming, the associations between higher maternal $\mathrm{HbA}_{1 \mathrm{c}}$ and higher concentrations of glucose among offspring may simply represent shared genetic predisposition. However, studies in Pima Indians demonstrated that 


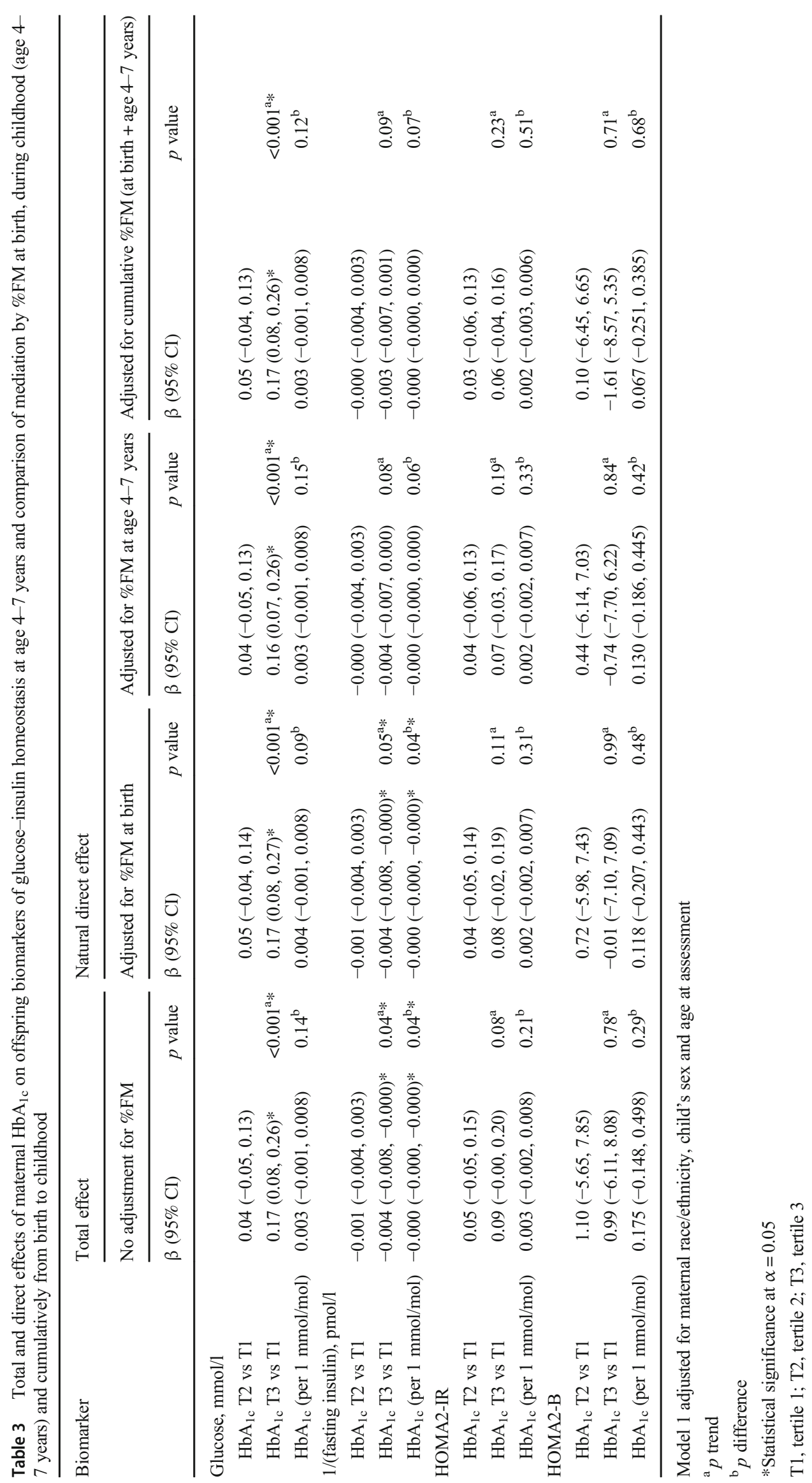


among siblings discordant for exposure to GDM, the likelihood of later developing diabetes was greater among the sibling exposed to the hyperglycaemic uterine environment, suggesting additional specific programming effects [36].

Strengths and limitations This study has several strengths. We used a state-of-the-art methodology for assessing offspring fat mass, whereas most previous studies $[4,6,23,30]$ used BMI, which represents both fat and FFM, or skinfolds, which are prone to measurement error. In addition, our sample included mother-offspring pairs from a diverse racial/ethnic background, thereby enhancing the generalisability of our findings. To our knowledge, our study is the first to examine cumulative adiposity from birth to early childhood (age 4-7 years) as mediating pathways underlying the relationship between in utero exposure to maternal blood glucose and offspring glucose-insulin homeostasis.

The current study is not without limitations. First, the Healthy Start Study at present may not be an ideal population in which to test lifecourse models, given the lack of temporal separation between adiposity assessment and the metabolic biomarkers during early childhood. Follow-up analyses with greater temporal separation between timing of adiposity measurement and assessment of the metabolic biomarkers are warranted. Second, maternal $\mathrm{HbA}_{1 \mathrm{c}}$ may be a lesssensitive biomarker of blood glucose level than fasting glucose; however, $\mathrm{HbA}_{1 \mathrm{c}}$ reflects glucose control over a longer period, so may be more relevant to in utero metabolic programming and long-term offspring outcomes. Third, given the low proportion of women with GDM in this analysis, it is possible that our null findings with respect to mediation by offspring adiposity could be due to a potential threshold effect, though previous studies among offspring exposed to more extreme levels of in utero hyperglycaemia also found that size at birth did not mediate offspring metabolic outcomes [37]. Fourth, as with all observational studies, we cannot exclude the possibility for residual confounding by unmeasured factors. However, we did not find large or concerning changes in the estimates of association, which is a typical sign that assumptions of mediation have been violated. Last, our study was conducted within a relatively small sample size. However, we do not think this hampered our ability to detect meaningful associations, given that findings from our study align with those from studies of over 4000 mother-offspring pairs $[3,6]$.

Conclusions and future directions Our primary finding was that children born to women in the highest vs lowest tertile of $\mathrm{HbA}_{1 \mathrm{c}}$ during early- to mid-pregnancy had $0.17 \mathrm{mmol} / \mathrm{l}$ higher fasting glucose at age 4-7 years. While this effect size is modest, it is worth noting that the Bogalusa Heart Study found that small differences in glucose during early life track into adulthood and that this is a better predictor of future diabetes risk than BMI $z$ score or change in BMI [38]. Further, among middle-aged non-diabetic adults, a $10 \mathrm{mg}$ / dl $(0.55 \mathrm{mmol} / \mathrm{l})$ difference in fasting glucose at baseline ( $\sim 3$ years of age) was associated with overt type 2 diabetes 7-8 years later [39]. Although our estimate is roughly one-third of that found among adults, estimates in the current study are likely of clinical relevance given the young age of the offspring, and prior studies indicating that these biomarkers track across life and are independent predictors of chronic disease risk [38]. The fact that we did not observe mediation by offspring adiposity contributes to ongoing efforts to identify targets for primordial prevention of chronic metabolic diseases [28]. Obesity plays a pathophysiological role in insulin resistance and dysglycaemia and, therefore, understanding the extent to which adiposity during early life stages mediates the relationship between in utero exposures and cardiometabolic health is of great interest. However, our results suggest the existence of alternative mechanistic pathways that operate beyond offspring adiposity. Future studies are required to determine whether this continues to be the case as children age. Complementary to continued follow-up in prospective cohorts are mechanistic studies nested within human cohorts [40,41], which have the potential to further explore biological pathways underlying observed epidemiological associations. These efforts will improve understanding of how maternal hyperglycaemia affects offspring glucose-insulin homeostasis, and whether there are critical time points during which interventions can reroute risk trajectories.

Acknowledgements We thank the Healthy Start II participants, as well as past and present research assistants at the Lifecourse Epidemiology of Adiposity and Diabetes (LEAD) Center, University of Colorado Denver Anschutz Medical Campus, Aurora, CO, USA.

Data availability The datasets analysed during the current study are available from the corresponding author on reasonable request.

Funding This study was funded by National Institute of Diabetes and Digestive and Kidney Diseases 5R01DK076648-10. ECF is supported by a T32 fellowship granted to the University of Colorado from the National Institute of Child Health and Human Development (5T32HD007186-39). WP is supported by the Center for Clinical and Translational Sciences Institute (CCTSI) KL2-TR002534.

Authors' relationships and activities The authors declare that there are no relationships or activities that might bias, or be perceived to bias, their work.

Contribution statement ECF, WP and DD conceived the research question. ECF conducted the analysis, wrote the initial draft of the paper and incorporated co-author comments. BMR curated the data, provided feedback on the analysis, and critically reviewed the manuscript. KAS, DD and WP contributed to data interpretation and reviewed the manuscript. All co-authors approved the final version of the paper. ECF and WP are the guarantors of this work and, as such, had full access to all the data in the study and take 
responsibility for the integrity of the data and the accuracy of the data analysis.

\section{References}

1. Lowe WL Jr, Lowe LP, Kuang A et al (2019) Maternal glucose levels during pregnancy and childhood adiposity in the Hyperglycemia and Adverse Pregnancy Outcome Follow-up Study. Diabetologia 62(4):598-610. https://doi.org/10.1007/ s00125-018-4809-6

2. Crume TL, Shapiro AL, Brinton JT et al (2015) Maternal fuels and metabolic measures during pregnancy and neonatal body composition: the healthy start study. J Clin Endocrinol Metab 100(4):16721680

3. Scholtens DM, Kuang A, Lowe LP et al (2019) Hyperglycemia and Adverse Pregnancy Outcome Follow-up Study (HAPO FUS): maternal glycemia and childhood glucose metabolism. Diabetes Care 42(3):381-392. https://doi.org/10.2337/dc18-2021

4. Bush NC, Chandler-Laney PC, Rouse DJ, Granger WM, Oster RA, Gower BA (2011) Higher maternal gestational glucose concentration is associated with lower offspring insulin sensitivity and altered beta-cell function. J Clin Endocrinol Metab 96(5):E803-E809. https://doi.org/10.1210/jc.2010-2902

5. Hapo Study Cooperative Research Group, Metzger BE, Lowe LP et al (2008) Hyperglycemia and adverse pregnancy outcomes. N Engl J Med 358(19):1991-2002. https://doi.org/10.1056/ NEJMoa0707943

6. Tam WH, Ma RCW, Ozaki R et al (2017) In utero exposure to maternal hyperglycemia increases childhood cardiometabolic risk in offspring. Diabetes Care 40(5):679-686. https://doi.org/10.2337/ dc16-2397

7. Li M, Hinkle SN, Grantz KL et al (2020) Glycaemic status during pregnancy and longitudinal measures of fetal growth in a multiracial US population: a prospective cohort study. Lancet Diabetes Endocrinol 8(4):292-300. https://doi.org/10.1016/S2213-8587(20) 30024-3

8. Hivert MF, Rifas-Shiman SL, Gillman MW, Oken E (2016) Greater early and mid-pregnancy gestational weight gains are associated with excess adiposity in mid-childhood. Obesity (Silver Spring) 24(7):1546-1553. https://doi.org/10.1002/oby.21511

9. Ben-Shlomo Y, Cooper R, Kuh D (2016) The last two decades of life course epidemiology, and its relevance for research on ageing. Int J Epidemiol 45(4):973-988. https://doi.org/10.1093/ije/dyw096

10. Harrod CS, Fingerlin TE, Chasan-Taber L, Reynolds RM, Glueck DH, Dabelea D (2015) Exposure to prenatal smoking and early-life body composition: the healthy start study. Obesity (Silver Spring) 23(1):234-241. https://doi.org/10.1002/oby.20924

11. Starling AP, Brinton JT, Glueck DH et al (2015) Associations of maternal BMI and gestational weight gain with neonatal adiposity in the Healthy Start study. Am J Clin Nutr 101(2):302-309. https:// doi.org/10.3945/ajen.114.094946

12. Singh B, Saxena A (2010) Surrogate markers of insulin resistance: a review. World J Diabetes 1(2):36-47. https://doi.org/10.4239/wjd. v1.i2.36

13. Levy JC, Matthews DR, Hermans MP (1998) Correct homeostasis model assessment (HOMA) evaluation uses the computer program. Diabetes Care 21(12):2191-2192. https://doi.org/10.2337/diacare. 21.12.2191

14. Ma G, Yao M, Liu Y et al (2004) Validation of a new pediatric airdisplacement plethysmograph for assessing body composition in infants. Am J Clin Nutr 79(4):653-660. https://doi.org/10.1093/ ajen/79.4.653
15. Fomon SJ, Haschke F, Ziegler EE, Nelson SE (1982) Body composition of reference children from birth to age 10 years. Am J Clin Nutr 35(5):1169-1175. https://doi.org/10.1093/ajcn/35.5.1169

16. Fields DA, Allison DB (2012) Air-displacement plethysmography pediatric option in 2-6 years old using the four-compartment model as a criterion method. Obesity 20(8):1732-1737. https://doi.org/10. 1038/oby. 2012.28

17. Oken E, Kleinman KP, Rich-Edwards J, Gillman MW (2003) A nearly continuous measure of birth weight for gestational age using a United States national reference. BMC Pediatr 3(1):6

18. Valeri L, Vanderweele TJ (2013) Mediation analysis allowing for exposure-mediator interactions and causal interpretation: theoretical assumptions and implementation with SAS and SPSS macros. Psychol Methods 18(2):137-150. https://doi.org/10.1037/ a0031034

19. Perng W, Mora-Plazas M, Marín C, Rozek LS, Baylin A, Villamor E (2013) A prospective study of LINE-1DNA methylation and development of adiposity in school-age children. PLoS One 8(4): e62587. https://doi.org/10.1371/journal.pone.0062587

20. Dabelea D, Harrod CS (2013) Role of developmental overnutrition in pediatric obesity and type 2 diabetes. Nutr Rev 71(Suppl 1):S62S67

21. Boeke CE, Oken E, Kleinman KP, Rifas-Shiman SL, Taveras EM, Gillman MW (2013) Correlations among adiposity measures in school-aged children. BMC Pediatr 13:99

22. Catalano PM, Farrell K, Thomas A et al (2009) Perinatal risk factors for childhood obesity and metabolic dysregulation. Am J Clin Nutr 90(5):1303-1313. https://doi.org/10.3945/ajcn.2008.27416

23. Krishnaveni GV, Veena SR, Hill JC, Kehoe S, Karat SC, Fall CH (2010) Intrauterine exposure to maternal diabetes is associated with higher adiposity and insulin resistance and clustering of cardiovascular risk markers in Indian children. Diabetes Care 33(2):402-404. https://doi.org/10.2337/dc09-1393

24. Lowe WL Jr, Scholtens DM, Lowe LP et al (2018) Association of gestational diabetes with maternal disorders of glucose metabolism and childhood adiposity. JAMA 320(10):1005-1016. https://doi. org/10.1001/jama.2018.11628

25. Skyler JS, Bakris GL, Bonifacio E et al (2017) Differentiation of diabetes by pathophysiology, natural history, and prognosis. Diabetes 66(2):241-255. https://doi.org/10.2337/db16-0806

26. Kelstrup L, Damm P, Mathiesen ER et al (2013) Insulin resistance and impaired pancreatic $\beta$-cell function in adult offspring of women with diabetes in pregnancy. J Clin Endocrinol Metab 98(9):37933801. https://doi.org/10.1210/jc.2013-1536

27. Guh DP, Zhang W, Bansback N, Amarsi Z, Birmingham CL, Anis $\mathrm{AH}$ (2009) The incidence of co-morbidities related to obesity and overweight: a systematic review and meta-analysis. BMC Public Health 9(1):88

28. Dabelea D (2018) Diabetes in youth-looking backwards to inform the future: Kelly West Award Lecture 2017. Diabetes Care 41(2): 233-240. https://doi.org/10.2337/dci17-0031

29. Simmonds M, Llewellyn A, Owen CG, Woolacott N (2016) Predicting adult obesity from childhood obesity: a systematic review and meta-analysis. Obes Rev 17(2):95-107. https://doi. org/10.1111/obr.12334

30. Lee AJ, Hiscock RJ, Wein P, Walker SP, Permezel M (2007) Gestational diabetes mellitus: clinical predictors and long-term risk of developing type 2 diabetes: a retrospective cohort study using survival analysis. Diabetes Care 30(4):878-883. https://doi.org/10. 2337/dc06-1816

31. Nolan CJ, Prentki M (2019) Insulin resistance and insulin hypersecretion in the metabolic syndrome and type 2 diabetes: time for a conceptual framework shift. Diab Vasc Dis Res 16(2):118-127

32. Aerts L, Sodoyez-Goffaux F, Sodoyez JC, Malaisse WJ, Van Assche FA (1988) The diabetic intrauterine milieu has a longlasting effect on insulin secretion by B cells and on insulin uptake 
by target tissues. Am J Obstet Gynecol 159(5):1287-1292. https:// doi.org/10.1016/0002-9378(88)90465-6

33. Aerts L, Van Assche FA (2006) Animal evidence for the transgenerational development of diabetes mellitus. Int J Biochem Cell Biol 38(5):894-903

34. Han J, Xu J, Long YS, Epstein PN, Liu YQ (2007) Rat maternal diabetes impairs pancreatic beta-cell function in the offspring. Am J Physiol Endocrinol Metab 293(1):E228-E236

35. Brumbaugh DE, Tearse $P$, Cree-Green $M$ et al (2013) Intrahepatic fat is increased in the neonatal offspring of obese women with gestational diabetes. J Pediatr 162(5):930-936.e931. https://doi. org/10.1016/j.jpeds.2012.11.017

36. Dabelea D, Hanson RL, Lindsay RS et al (2000) Intrauterine exposure to diabetes conveys risks for type 2 diabetes and obesity: a study of discordant sibships. Diabetes 49(12):2208. https://doi. org/10.2337/diabetes.49.12.2208

37. Blotsky AL, Rahme E, Dahhou M, Nakhla M, Dasgupta K (2019) Gestational diabetes associated with incident diabetes in childhood and youth: a retrospective cohort study. Can Med Assoc J 191(15): E410-E417. https://doi.org/10.1503/cmaj.181001

38. Nguyen QM, Srinivasan SR, Xu J-H, Chen W, Kieltyka L, Berenson GS (2010) Utility of childhood glucose homeostasis variables in predicting adult diabetes and related cardiometabolic risk factors. The Bogalusa Heart Study 33(3):670-675
39. Abdul-Ghani MA, Williams K, DeFronzo RA, Stern M (2007) What is the best predictor of future type 2 diabetes? Diabetes Care 30(6):1544-1548. https://doi.org/10.2337/dc06-1331

40. Boyle KE, Patinkin ZW, Shapiro ALB, Baker PR 2nd, Dabelea D, Friedman JE (2016) Mesenchymal stem cells from infants born to obese mothers exhibit greater potential for adipogenesis: the Healthy Start BabyBUMP Project. Diabetes 65(3):647-659. https://doi.org/10.2337/db15-0849

41. Boyle KE, Patinkin ZW, Shapiro ALB et al (2017) Maternal obesity alters fatty acid oxidation, AMPK activity, and associated DNA methylation in mesenchymal stem cells from human infants. Mol Metab 6(11):1503-1516. https://doi.org/10.1016/j.molmet.2017. 08.012

42. Institute of Medicine (2009) Weight gain during pregnancy: reexamining the guidelines. National Academies Press, Washington, DC

43. WHO Multicentre Growth Reference Study Group (2009) WHO Child Growth Standards: growth velocity based on weight, length and head circumference: methods and development. World Health Organization, Geneva

Publisher's note Springer Nature remains neutral with regard to jurisdictional claims in published maps and institutional affiliations. 